Doi: http://dx.doi.org/10.1590/1809-4430-Eng.Agric.v41n3p368-378/2021

\title{
PARAMETERS FOR MODELING PASSIVE SUSPENSIONS OF SPRAY BARS
}

\author{
Rafael Kappaun ${ }^{1}$, Agenor D. de Meira Junior', Márcio Walber ${ }^{\text {* }}$
}

${ }^{1 *}$ Corresponding author. University of Passo Fundo/ Passo Fundo - RS, Brazil.

Email: mwalber@upf.br | ORCID ID: https://orcid.org/0000-0002-4472-0937

\section{KEYWORDS}

Rigid body dynamics, finite elements, simulation, sprayer.

\begin{abstract}
The uniformity and quality of spraying depend on the stability of the spray boom, defined as the suspension system between the boom and the machine chassis. This paper presents a procedure for improving the performance of passive spray bar suspensions through parameter adjustment. Two multibody dynamics models of a tractor sprayer set were developed to evaluate their suspension systems: a rigid body dynamics model (RBDM) and a finite element model (FEM) using deformable bodies. To calibrate the models in the experiment, an accelerating force was applied to the suspension, and the displacements of the shock absorber and the rubber springs were monitored. The FEM is more suitable for the evaluation of the horizontal oscillations of the bar, based on root mean square (RMS) values and a standard curve used to evaluate the stability of the bar. The horizontal stiffness of the bar significantly influences the oscillatory displacement and must be included in the simulation models. Resizing the structure can reduce the horizontal oscillations of the bar.
\end{abstract}

\section{INTRODUCTION}

Spraying fields with uneven surfaces can cause the spray boom to oscillate vertically and horizontally. The oscillations, in turn, cause the spraying of the plants to be uneven, but a suspension mechanism between the spray bar and the machine chassis reduces its unwanted movement (Sobotka \& Lipinski 2015; Gohari et al., 2017). Figure 1 shows the coordinate system for the system used in this paper.

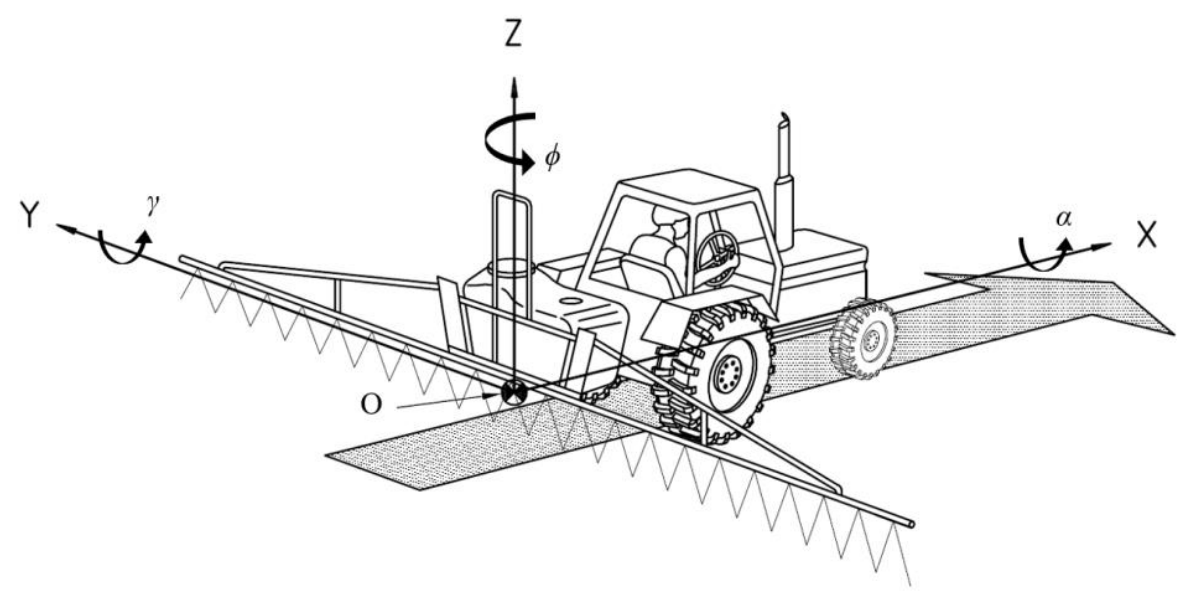

FIGURE 1. Generalized coordinates: tractor and bar movements.

\footnotetext{
${ }^{1}$ University of Passo Fundo/ Passo Fundo - RS, Brazil.

Area Editor: João Paulo Arantes Rodrigues da Cunha

Received in: $10-23-2020$

Accepted in: 4-20-2021
} 
Figure 1 shows how tire deflections caused by different field profiles on the left and right sides of the tractor result in vertical movements (translational movement along the $\mathrm{Z}$ axis) and rolling of the boom (rotation around the $\mathrm{X}$ axis, $\alpha$ ). Downward movement of the bar causes high concentrations of agro-defensive chemicals under the nozzles and a reduced application between nozzles because of the missing but necessary overlap of the spray cones. Upward movement results in a low liquid deposition under the most extreme nozzles; losses are associated with the drift effect. Figure 2 illustrates how vertical displacement and rolling affect spray deposition (Langenakens, 1999).

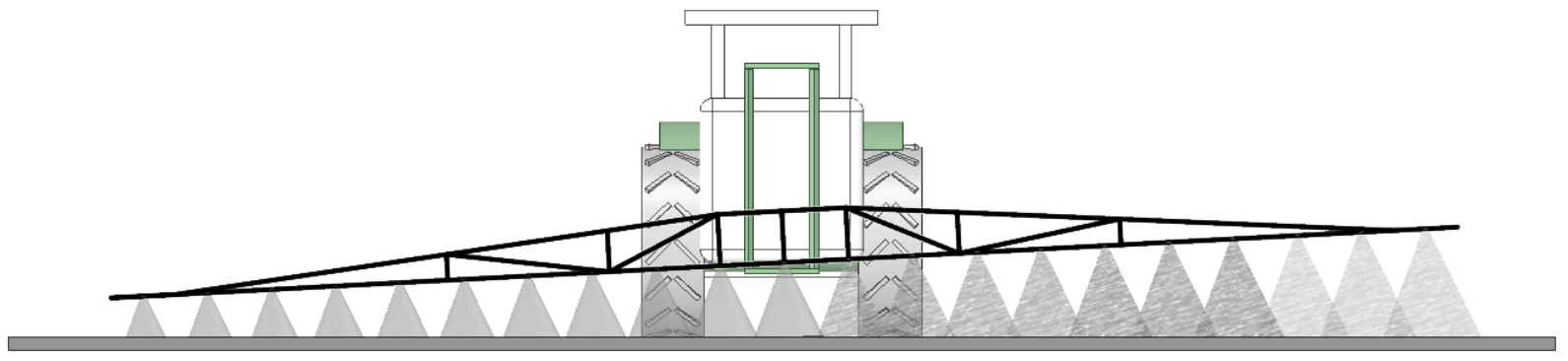

FIGURE 2. Influence of vertical oscillations.

Engelen (2008) defined spray bars as slender structures with high horizontal flexibility in the $\mathrm{X}$ direction and low structural damping. Resonance frequencies are low and quickly excited by field vibrations. In contrast to rigid vertical and rolling body motions, horizontal oscillations are caused by the deformation of the spray bar. The induced variations in the longitudinal velocity of the nozzle disturb the distribution pattern. Figure 3 shows how deformation of the boom structure creates local over- or underapplication of the sprayed liquid. As the design widths of spray booms continue to increase, reaching up to $50 \mathrm{~m}$, this resonance problem has become a critical issue.

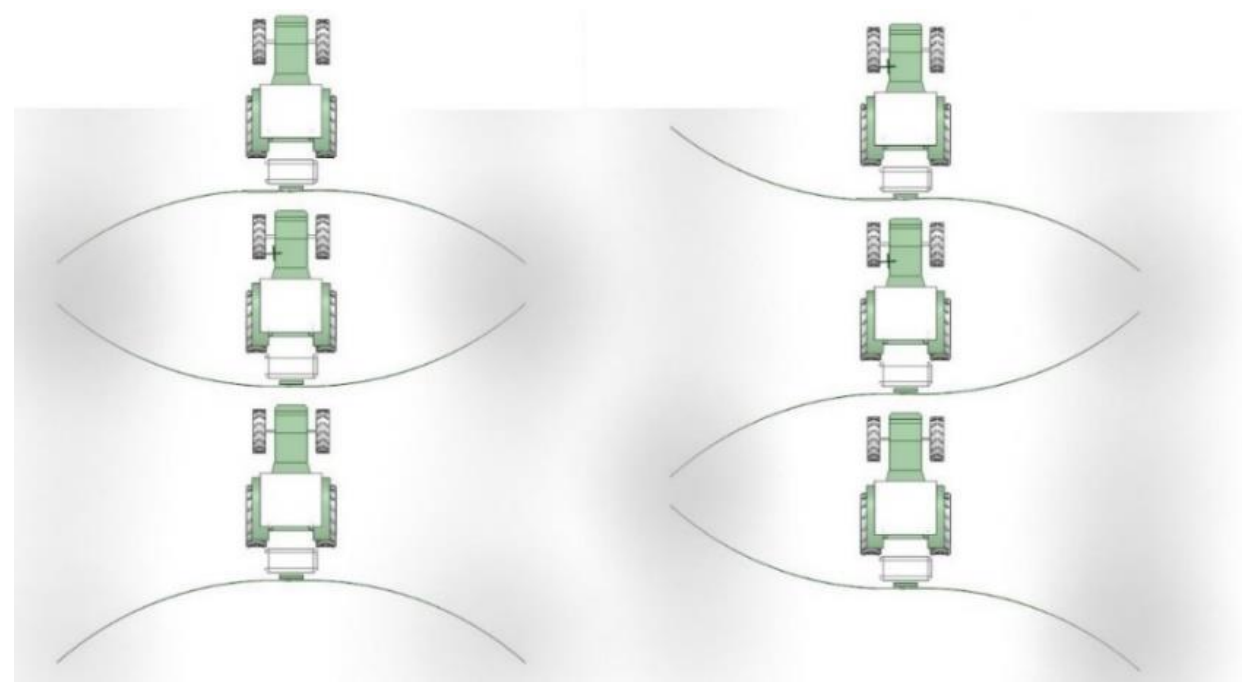

FIGURE 3. Influence of horizontal oscillations of the spray boom.

Thus, the smaller the oscillations of the spray bar, the more uniform the application. Machado et al. (2016) compared the vertical and horizontal oscillations of three different self-propelled sprayers marketed in Brazil and found statistical differences between the vertical and horizontal oscillations of the sprayers. This demonstrated that the vibration-absorbing systems, or suspensions, influence the spraying result.

According to Herbst et al. (2018), there is no standard method to test the accuracy of sprayer boom suspensions objectively. Different modeling techniques have been used to represent suspension systems and sprayer booms, such as those based on undeformable bodies (Pontelli et al., 2010 and 2009; Tahmasebi et al., 2018) and those based on deformable elements (Koc, 2015; Manea et al., 2018). Alternative modeling was presented by Bjornsson et al. (2013), with rigid segments connected by torsional springs to represent the horizontal stiffness of the bar.

The finite element method is a general approximation method for continuous systems, which can be applied regardless of the shape of the structure and the loading conditions. It allows stresses and strains to be calculated (Alves, 2013). The advantages and limitations of rigid body and finite element models have been discussed in the literature; however, no published scientific study compares the two techniques applied using the same tractor and sprayer settings.

This study presents the differences between the rigid body dynamics model (RBDM) and finite element model (FEM). The stiffness and damping parameters for a passive suspension system of spray bars are determined through a simulation model and discussed. 


\section{MATERIAL AND METHODS}

\section{Computational numerical model}

This study modeled the tractor with boom sprayers shown in Figure 4. The sprayer has a trapezoidal "A form" suspension weighing $560 \mathrm{~kg}$ and having an 800-liter reservoir and a spray bar $18 \mathrm{~m}$ wide. The $75 \mathrm{HP}$ tractor has $4 \times 4$ traction and a mass of $5500 \mathrm{~kg}$, including ballast. There are two 12.4-24 tires on the front axle, each with six plies. On the rear axle, a pair of tires is mounted on each side; each pair consists of a 13.6-38 with 14 plies and an 18.4-30 with ten plies.

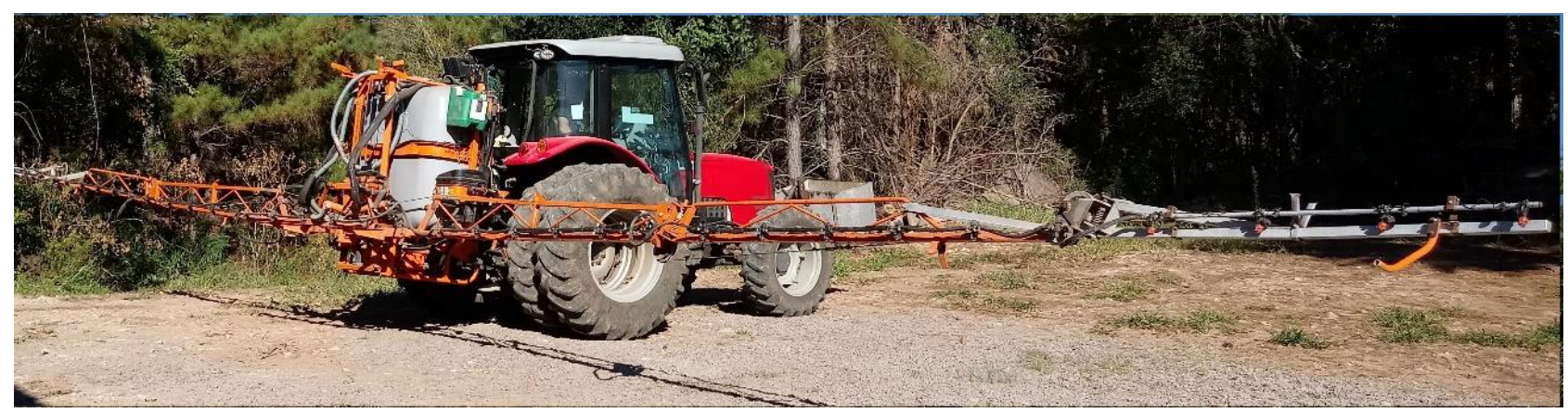

FIGURE 4. Tractor sprayer set used in the research.

Due to the complexity of this model, Ansys Workbench software was used to perform the simulations. The passive suspension of the sprayer boom is the principal concern of the research, so the model was kept simple, including the main mechanical components shown in Figure 5.

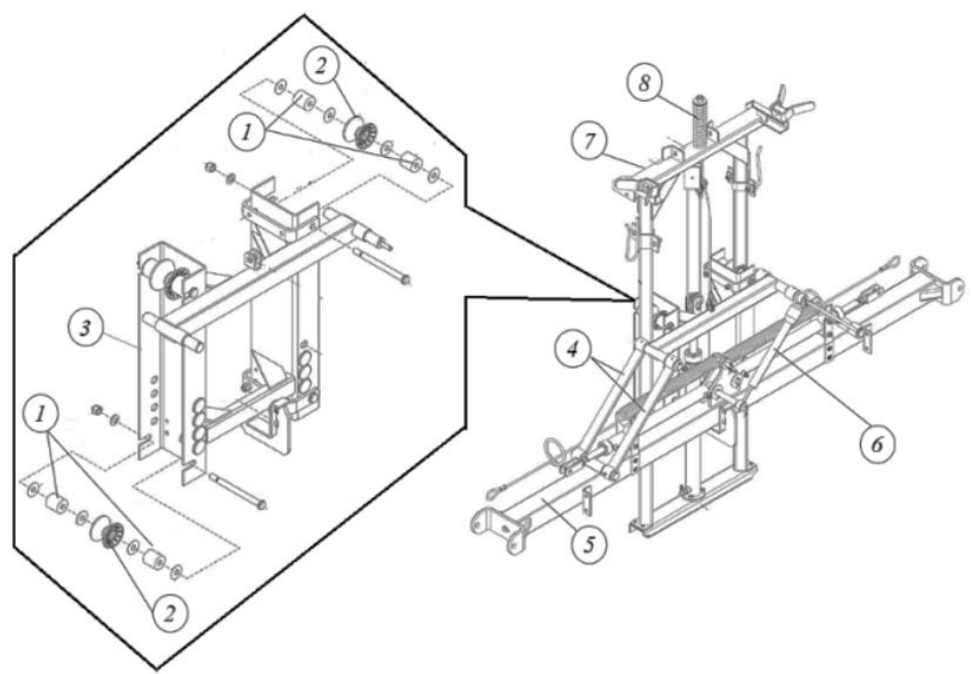

1 - Rubber springs

2 - Pulley

3 - Mobile board

4 - Connecting rods

5 - Oscillating frame

6 - Frame shock absorber

7 - Fixed board

8 - Coil spring

FIGURE 5. Passive A-form trapezoidal suspension.

For the tractor and sprayer set in Figure 5, the authors developed the virtual geometric model in Figure 6. Two types of models were developed for the dynamic simulation: one is the RBDM, in which all bodies are considered undeformable; the other is the FEM, a model consisting of finite elements, in which the bodies in gray in Figure 6 are considered undeformable, and the geometries highlighted by red are discretized with BEAM188 elements representing spray bar deformations.

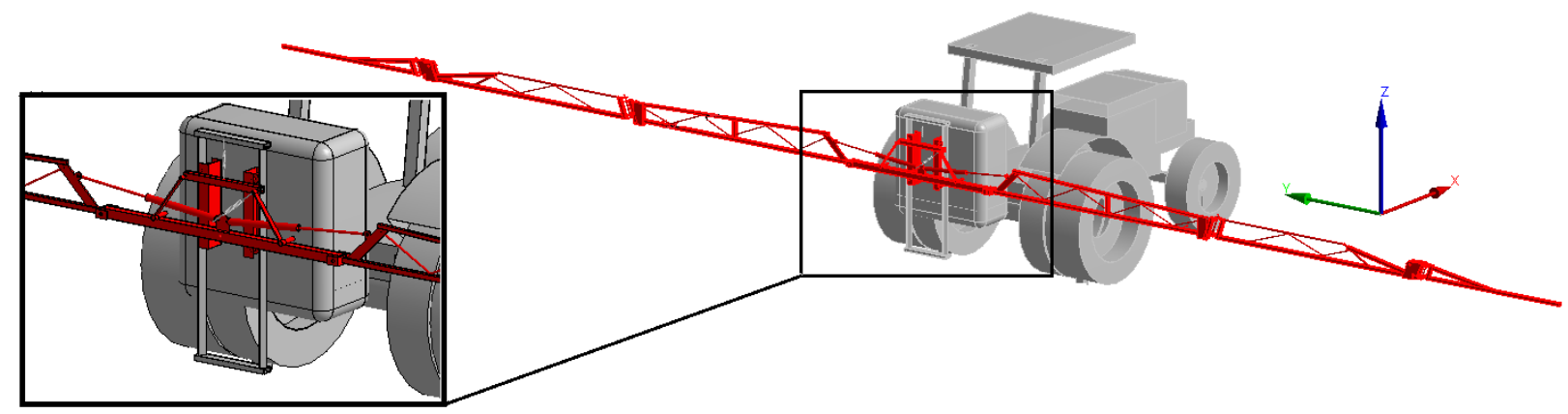

FIGURE 6. The geometric model used in the simulation. 
The roll of the bar that results in vertical oscillations at its ends is influenced by the frame shock absorber (item 6 in Figure 5, COFAP model 2210), mounted between the movable and oscillating frames. The shock absorber is nonlinear and has the characteristics shown in Figure $7 b$.

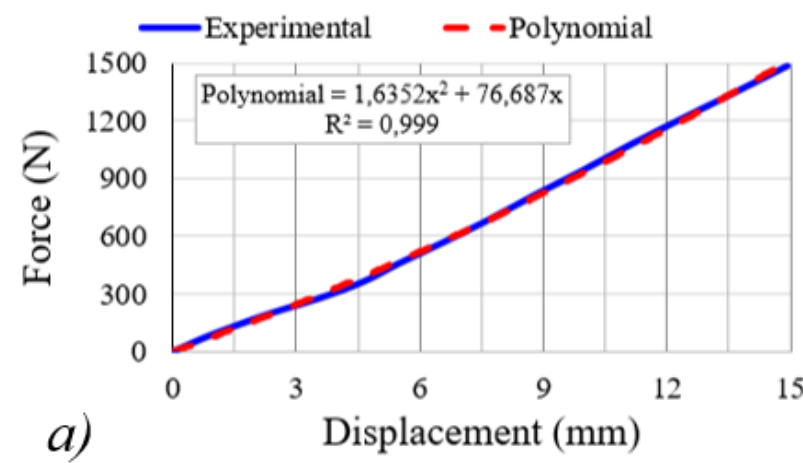

The horizontal movements are influenced by the rubber springs (item 1 in Figure 5), positioned between the movable and fixed frames. The rubber springs are nonlinear, and their curve can be represented by the polynomial function shown in Figure $7 a$.

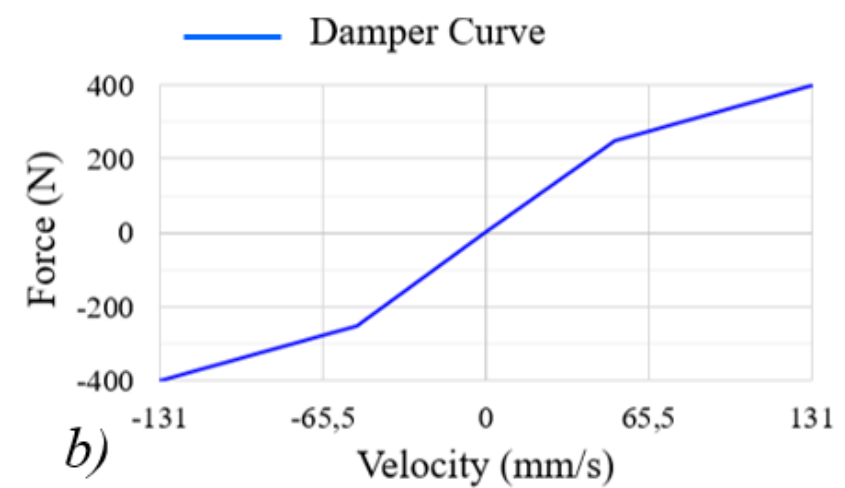

FIGURE 7. Passive suspension parameters: $a$ ) Curve of force $\times$ velocity of damper $2210 ; b$ ) Force $\times$ displacement graph of a rubber spring.

Schematically, the modeled equipment can be interpreted according to Figure 8. The tires are represented by equivalent linear springs with constant damping: $\mathrm{k} 1=$ 191.4 N/m, k2 = 944.8 N/m, k3=32.25 N/m, k4 = nonlinear curve of the damping springs, $\mathrm{C} 1=3 \mathrm{~N} \cdot \mathrm{m} / \mathrm{s}, \mathrm{C} 2=$ Curve of the frame damper, and $\mathrm{k} 1$, equivalent to the pair of tires used on the rear axle.
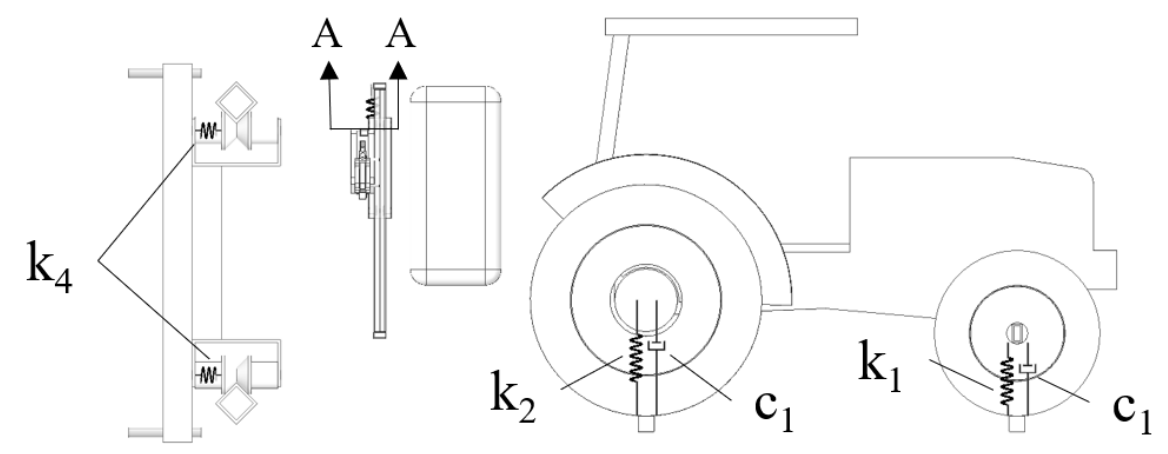

$\mathrm{C}_{1}$

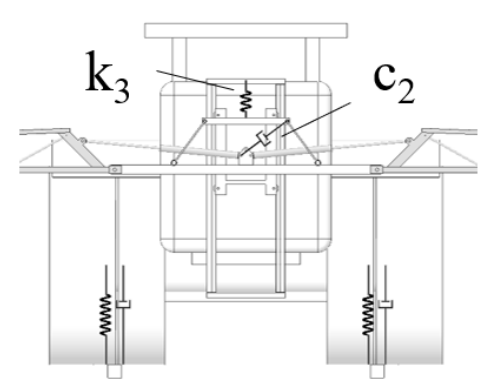

FIGURE 8. Schematic representation of springs and dampers.

RBDM and FEM are used in the simulation of two free vibration tests for parameter calibration. After being calibrated, they are subjected to a smooth track profile 100 $\mathrm{m}$ long and according to ISO 5008. The time required to run this track is 73.175 seconds. The normed profile provides an international standard with an excitation source that can be used in agricultural machinery calculations (Tahmasebi et al., 2018; Pontelli et al., 2010; Stãnescu et al., 2010).

In all simulations, the acceleration of gravity is applied to compute the weights of components.

\section{Experimental procedure}

The experimental tests provided data to calibrate the models. To read and store the measured information, we used the HBM Quantum X data acquisition system, two
HBM WA200-L LVDTs, and a Silicon Designs 2460-010 accelerometer. The data were collected with an acquisition rate of $1000 \mathrm{~Hz}$, and the Catman Easy software was used for post-processing.

One LVDT was used to monitor the deformation of one of the rubber springs, which results in horizontal oscillations at the end of the bar. Another LVDT was used to monitor the relative displacement between the rod and cylinder of the frame shock absorber, which results in vertical oscillations at the end of the bar. The accelerometer is fixed to the base of the fixed frame to acquire the horizontal $(X)$ and vertical $(Z)$ accelerations at a point on the machine chassis independent of the suspension and bar. Figure 9 shows the mounting locations of the LVDTs and the accelerometer. 


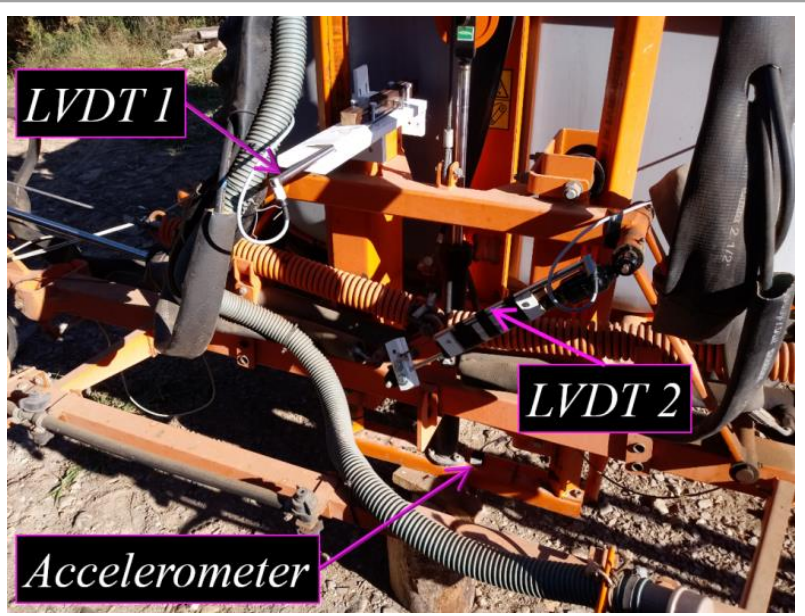

FIGURE 9. Mounting locations for the accelerometer and LVDTs.

The experiment was performed in two stages. The first stage identified the Coulomb damping through two free vibration tests: one for calibration corresponding to the rolling motion (rotation around the $\mathrm{X}$-axis, $\alpha$ ); and the other for calibration corresponding to the yaw motion (rotation around the Z-axis, $\phi$ ).

In the free vibration tests, the end of the bar was displaced a distance corresponding to the roll and yaw modes; then, the structure was released and allowed to vibrate freely. The simulation models were adjusted by adding damping to approximate the performance difference between the simulated and experimental results.

The second stage of experimental data collection consisted of moving the tractor and sprayer together at 6 $\mathrm{km} / \mathrm{h}$ in a field with an unknown random profile for $235 \mathrm{~m}$. The test started at $28.57378^{\circ} \mathrm{S}$ latitude, $51.85071^{\circ} \mathrm{W}$ longitude, and $585.85 \mathrm{~m}$ altitude and ended at $28.57587^{\circ} \mathrm{S}$ latitude, $51.85052^{\circ} \mathrm{W}$ longitude, and of $587.79 \mathrm{~m}$ altitude. The surface of the field is generally smooth with few potholes. Data collection followed the ISO 14131 recommendation.

With the aid of a static profilometer, two sections of the random field profile were measured, one $15 \mathrm{~m}$ long (adopted as the reference) and another $8 \mathrm{~m}$ long, including the largest hole in the $235 \mathrm{~m}$ path. Both profiles were measured with a displacement increment of $0.5 \mathrm{~m}$. Figure 10 presents the two sections measured over the first $15 \mathrm{~m}$ of the smooth profile per ISO 5008 .

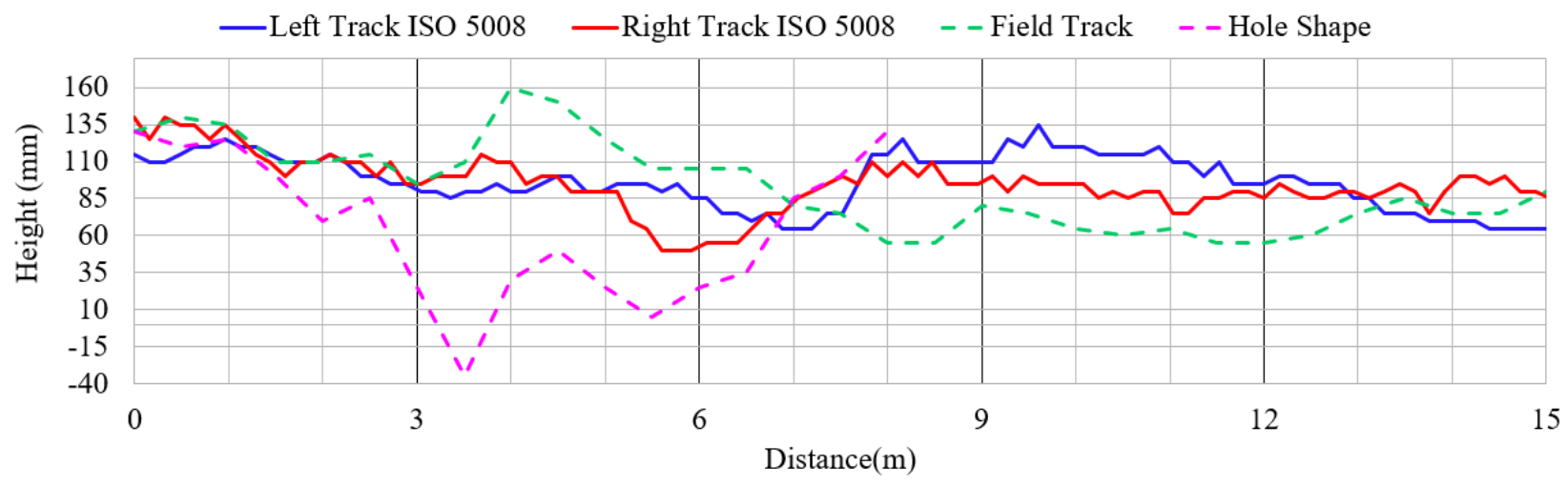

FIGURE 10. Standardized and measured track profiles.

\section{Model calibration}

The free rolling and yawing vibration tests were reproduced in a virtual environment to calibrate the suspensions of the numerical RBDM and FEM.

It was necessary to add a nonlinear damping element in parallel with the frame damper to adjust for the rolling motion. Moreover, to adjust for the yaw movement, it was necessary to add a damping element for each rubber spring.
As pointed out by Pontelli (2009), the Coulomb friction significantly influenced the results of the numerical models because it was necessary to consider the losses by friction.

Figure 11 shows the experimental results of the relative displacement between the rod and cylinder of the damper measured by LVDT 2 for the numerical RBDM and FEM. It can be seen that the numerical models represented the behavior of the damper accurately. 


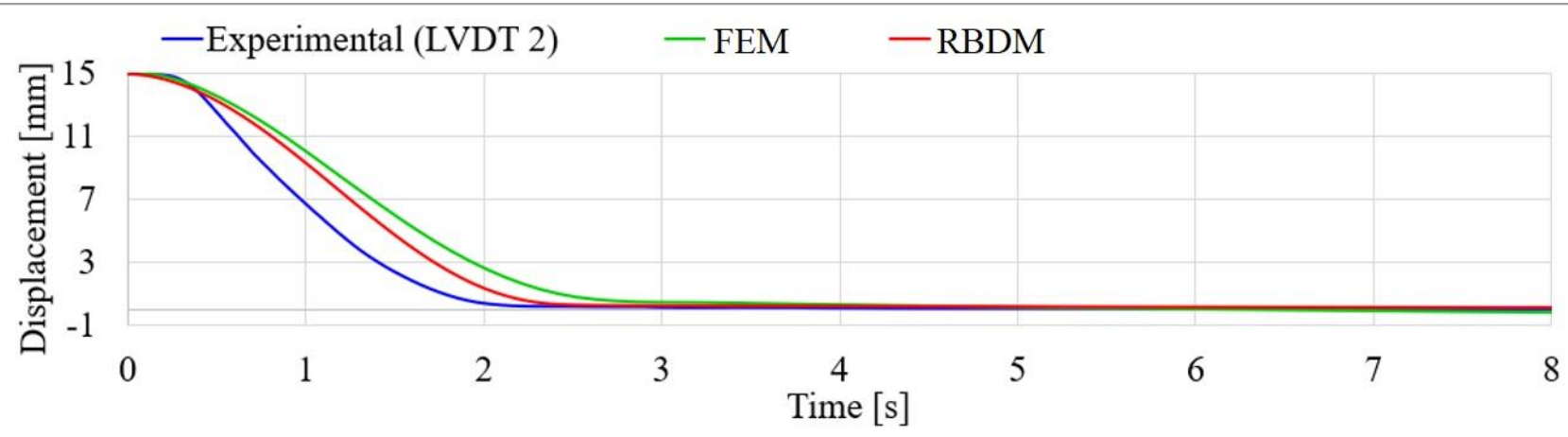

FIGURE 11. Experimental $\times$ numerical result of the relative motion of the damper in the rolling test.

Figure 12 presents the experimental results of the rubber spring deformation measured by LVDT 1 for the numerical RBDM and FEM.

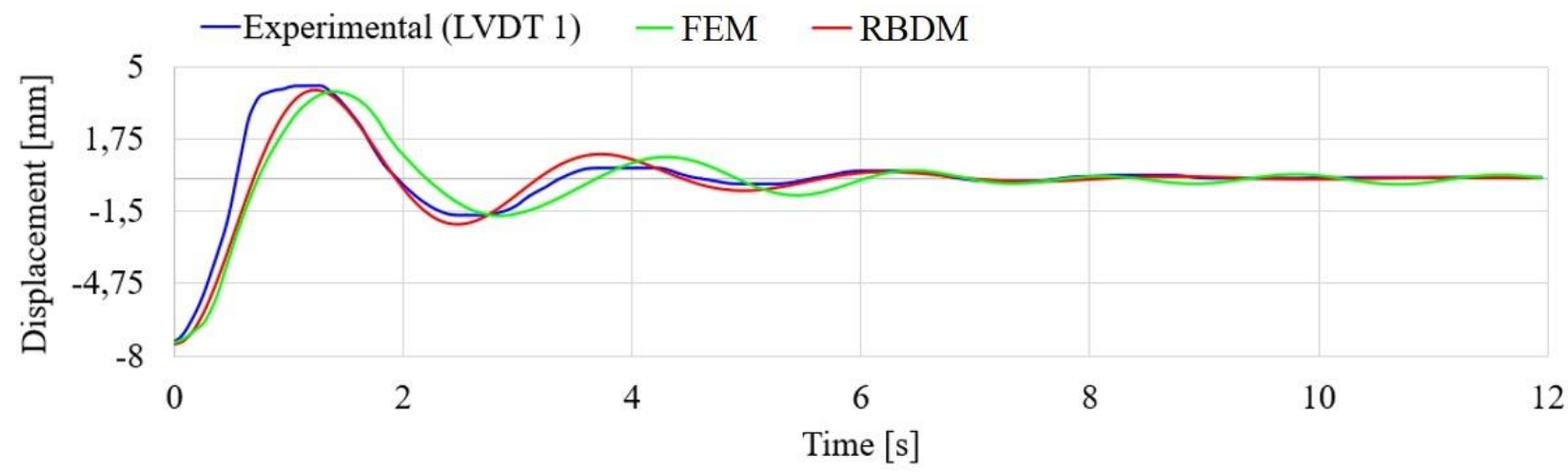

FIGURE 12. Experimental $\times$ numerical comparison of rubber spring deformation in yaw test.

The average RMS of each curve was used to compare the curves presented in Figures 11 and 12 quantitatively; the values are given in Table 1 . The maximum difference found in the FEM for the rolling mode was $25.98 \%$.
The FEM produced more differences from the experimental results because only the damping was adjusted for calibration. Spring stiffness and mass could have been changed in calibration, but it was decided to keep these two characteristics the same in both models.

TABLE 1. RMS differences between experimental and numerical results in free vibration tests.

\begin{tabular}{ccccc}
\hline Curve & \multicolumn{2}{c}{ Free Rolling Vibration } & \multicolumn{2}{c}{ Slewing free vibration } \\
Difference & RMS & - & RMS & - \\
Experimental & 5,20 & $13,65 \%$ & 1,65 & $-9,69 \%$ \\
RBDM & 5,91 & $25,96 \%$ & 1,49 & 1,89 \\
FEM & 6,55 & & $1,54 \%$ \\
\hline
\end{tabular}

\section{RESULTS AND DISCUSSION}

Once the simulation models were calibrated, the suspension performance was evaluated by subjecting it to the smooth track profile provided by ISO 5008. Figures 13 and 14 show the signals in the frequency domain, vertical and horizontal, respectively, with the FFTs of the experimental accelerations and the numerical RBDM and FEM.
In the FFTs, a qualitative coherence between the numerical and experimental results was noticed because of the vertical excitations concentrated below $6 \mathrm{~Hz}$, as shown in Figure 13. The horizontal excitations are below $4 \mathrm{~Hz}$ (Figure 14). It is possible to confirm that the normed profile produces similar behavior to the experimental one over the range of exciting frequencies. 


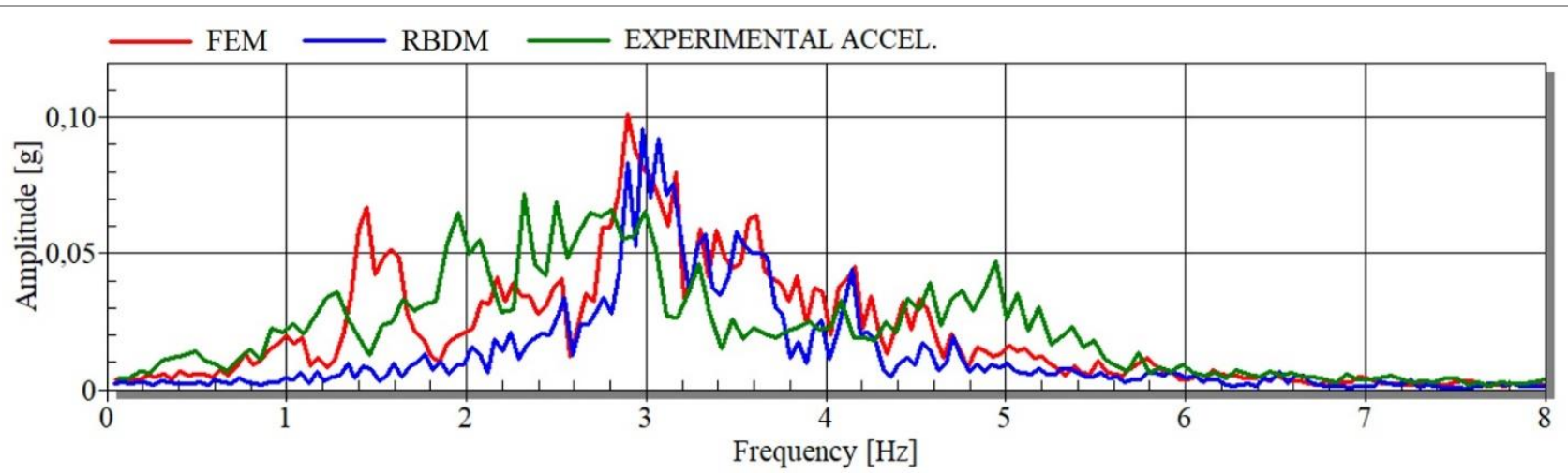

FIGURE 13. Experimental numerical vertical FFTs corresponding to accelerometer data.

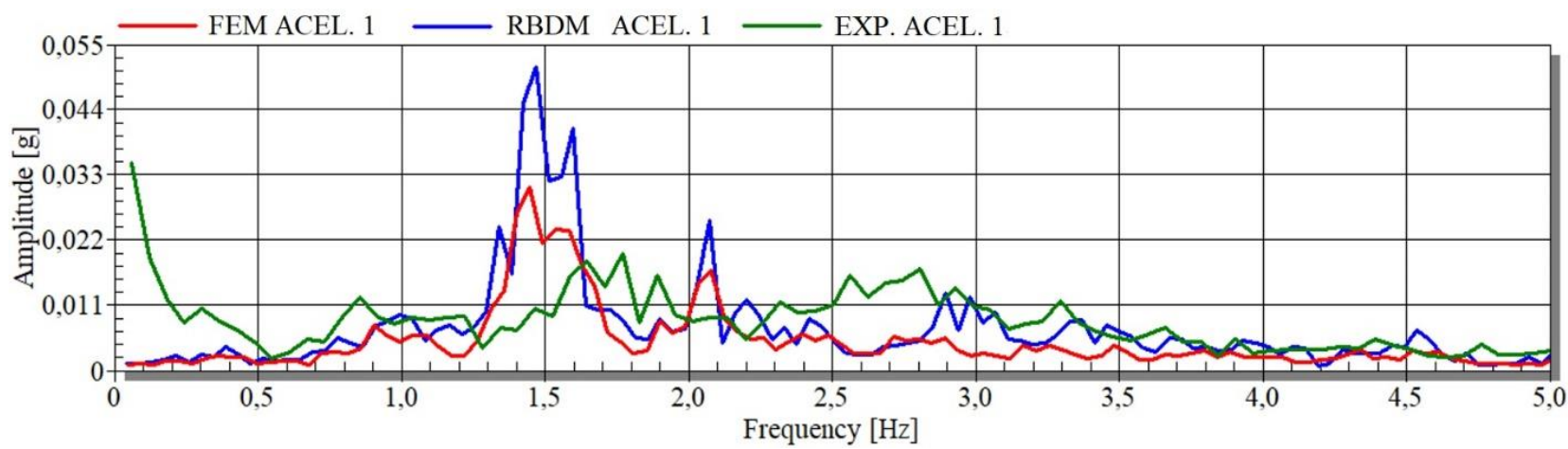

FIGURE 14. Experimental numerical horizontal FFTs corresponding to accelerometer data.

The magnitude of the accelerations obtained numerically cannot be compared directly with those obtained experimentally. The field profile is random and different from the normed one, as indicated in Figure 10.

Figures 15 and 16 show the FFTs of the displacement path with the numerical RBDM and FEM corresponding to LVDT 1 and LVDT 2 measurements, respectively. Using the experimental FFTs, the natural frequencies of the suspension of $0.12 \mathrm{~Hz}, 0.24 \mathrm{~Hz}$, and 0.36 $\mathrm{Hz}$ were identified. Both simulation models had responses close to these frequencies. Larger amplitudes below $1.0 \mathrm{~Hz}$ were observed in the experimental data, suggesting that the field profile is more severe in this range.

In Figure 15, a greater displacement was noted in the RBDM than in the FEM because the RBDM responded only with rigid body movements, as can be seen in the frequency range near $1.5 \mathrm{~Hz}$. In contrast, the FEM has more vibration modes and responds over more frequencies with lower amplitudes.

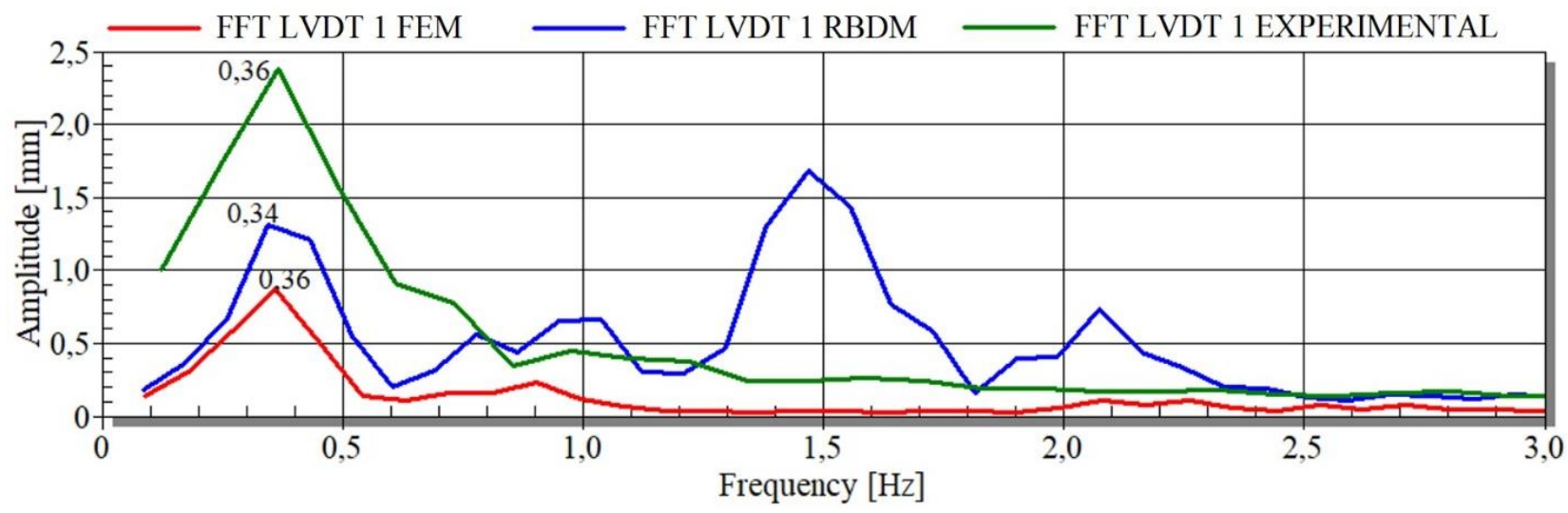

FIGURE 15. Experimental and numerical results for the rubber spring, LVDT 1. 


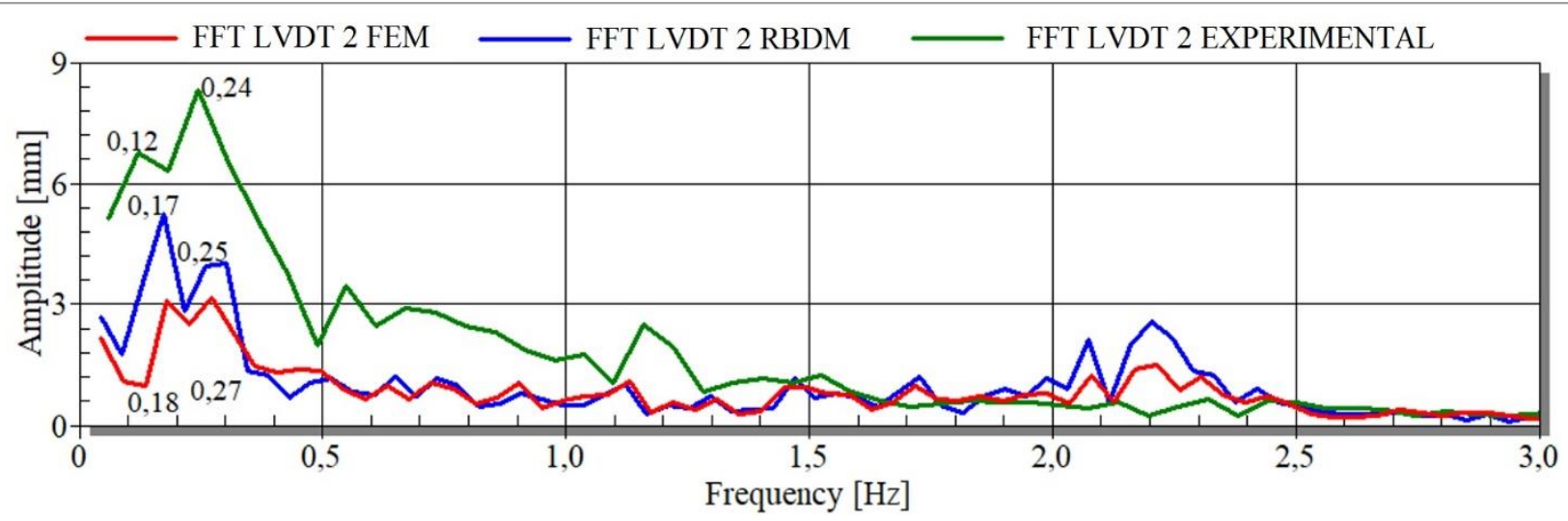

FIGURE 16. Experimental and numerical results for the frame damper, LVDT 2.

Figures 17 and 18 show that the vertical and horizontal displacements occurred at the left end of the spray bar. The FEM produced greater amplitudes due to the sum of the rigid body motion and the deformation of the structure. It is possible that the displacement due to the deformation of the structure acted opposite to the direction of rigid body movement and reduced the amplitude of the movement. This effect was highlighted in the first seconds of the vertical displacement graph (Figure 17) when the RBDM showed a more significant negative displacement.

At various points on the FEM curve in Figure 18, the amplitudes of the FEM are more than twice those of the RBDM. This situation was not observed in the vertical displacements (Figure 17) since the structure's vertical stiffness was greater. A more rigid structure would yield closer results between the models.

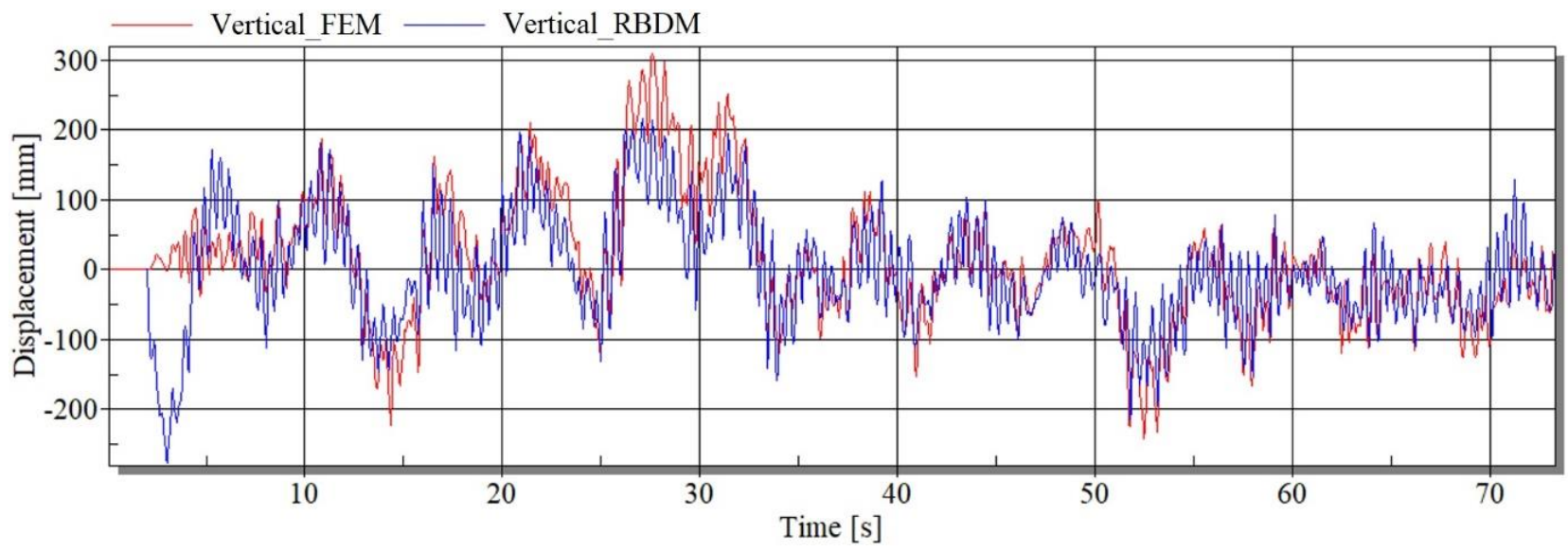

FIGURE 17. Vertical displacement at the left end of the spray bar.

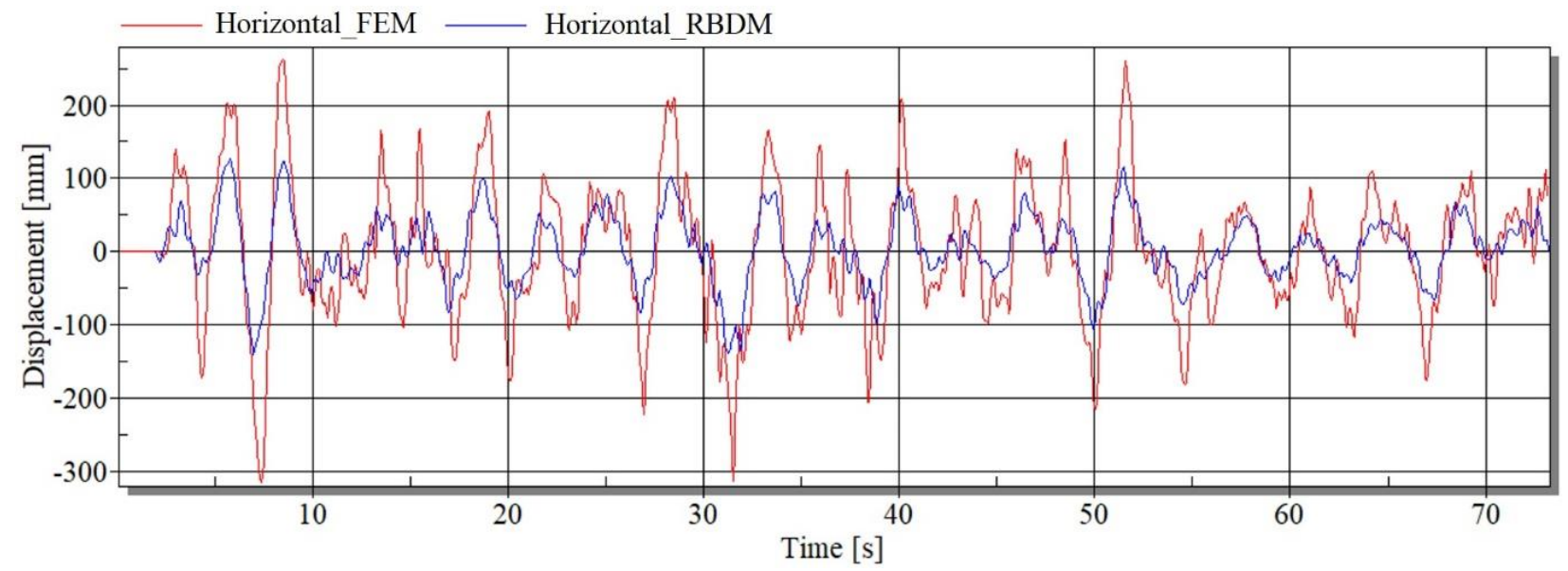

FIGURE 18. Horizontal displacement at the left end of the spray bar.

Corroborating the observations of Engel (2008) and Manea et al. (2018), the authors found that the horizontal deformations of the bars contributed to increased oscillation amplitudes. However, the rigid-body motions of the suspension cannot be neglected in this orientation.
In Figures 17 and 18, the behaviors of the FEM and RBDM are similar because of the similar rigid body motions allowed by adjusting the bar suspension to respond to free vibration in the tests. 
The FEM is more applicable for evaluating the performance of a suspension system for spray bars because it considers the deformation of the structure in the result. The RBDM can guide the development of a design to approximate the ideal values of stiffness, damping, and construction of a suspension system. A simpler model of suspension system behavior is better used to deal with the increasing complexity of the suspension system and variants. After a concept has been defined, an FEM can be developed to improve the suspension.

Next, this paper presents a procedure using the finite element simulation model to make design changes for mitigating the vibrations transmitted to the spray bar. To this end, the stiffness parameters of the rubber springs and the damping of the frame are altered.

The bar suspension's initial stiffness and damping parameters were multiplied by factors of 0.5 and 1.5 . The configuration presented in the previous section will be denominated original design state.

It should be noted that the frame damper affects only vertical oscillations at the end of the boom and that the rubber springs affect only the horizontal oscillations at the end of the spray bar.

The three simulated stiffness and damping levels resulted in five simulation models. Table 2 indicates the simulated stiffness and damping combinations by an X.

TABLE 2. Simulated stiffness and damping combinations.

\begin{tabular}{cccc}
\hline Variants & Damping x 0.5 & Original Dampening (x 1) & Damping x 1.5 \\
\hline Stiffness x 0.5 & & $\mathrm{X}$ & $\mathrm{X}$ \\
Original Stiffness (x 1) & $\mathrm{X}$ & $\mathrm{X}$ & $\mathrm{X}$ \\
Stiffness x 1.5 & & $\mathrm{C}$ & \\
\hline
\end{tabular}

Figure 19 presents the vertical oscillations at the left end of the spray bar over a range of 33 to 43 seconds, influenced by three damping levels, maintaining the original rubber spring stiffness. The simple graphics do not determine which damping level provides the best stability for the spraying bar.

The average value can be helpful to understand the behavior of the bar: if the bar stays longer close to the ground, a negative value is indicated; if further above the ground, it is positive. However, an average cannot be used to judge the performance of a suspension system. A system with excessively large positive and negative amplitudes, and undesirable behavior for spraying, could have a zero average.

This is why the RMS value is more suitable to evaluate the suspension performance. It is a statistical measure of the magnitude of a variable quantity reflecting equivalence with a continuous signal; therefore, the smaller the RMS value, the more stable the spray boom.

Table 3 shows the maximum and minimum amplitudes and the RMS value, with better stability (lower RMS values) for damping $\times 1.5$.

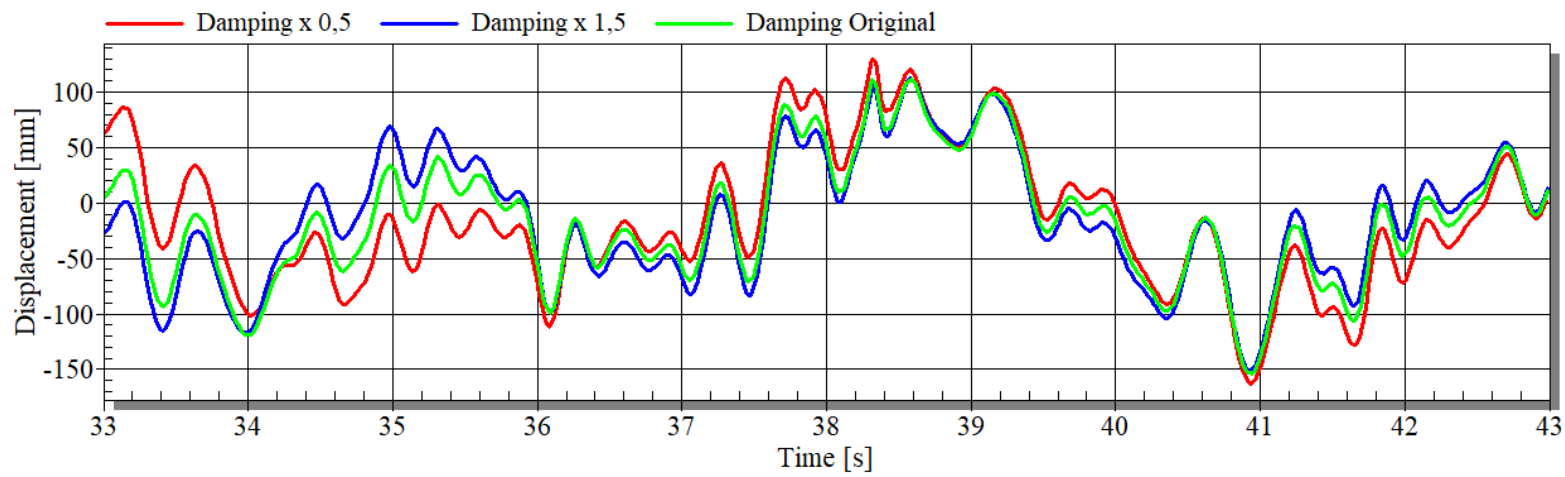

FIGURE 19. Vertical displacement of the end of the bar in the FEM.

Figure 20 presents 7 to 17 seconds of horizontal oscillations at the left end of the spray bar, influenced by three levels of rubber spring stiffness, and maintaining the original damping level. As had occurred in evaluating the damping level, the determination of the best stiffness level is not visible in a graph in the time domain.

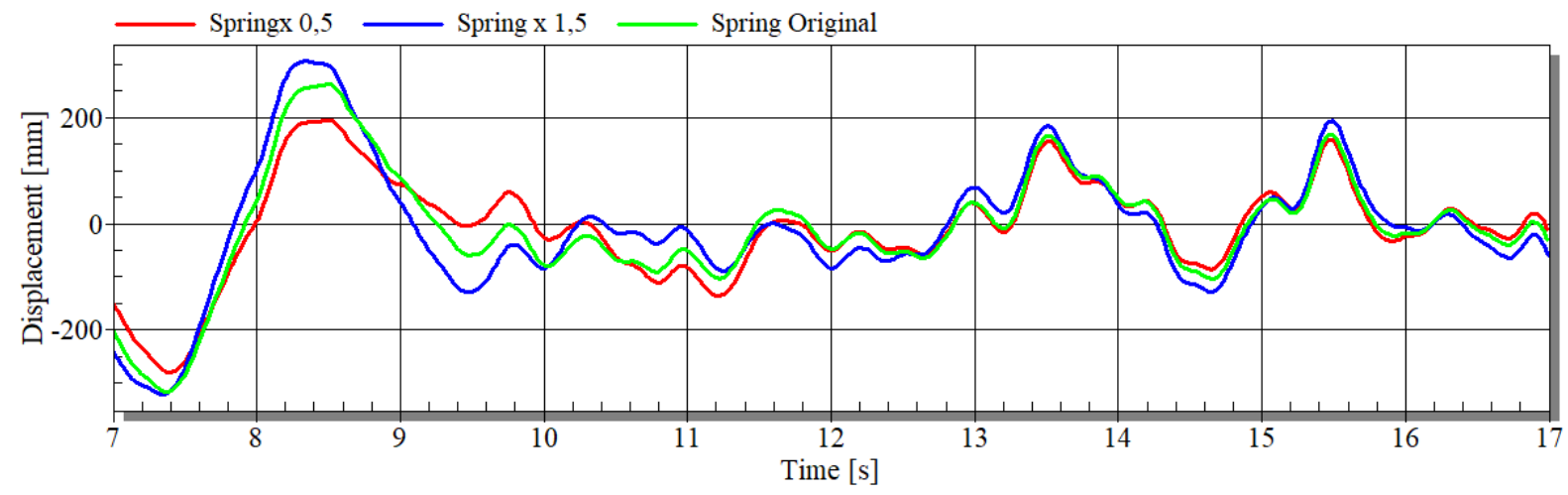

FIGURE 20. Horizontal displacement of the end of the bar in the FEM. 
Table 3 compares the maximum and minimum horizontal displacements and the average RMS of the horizontal oscillations, observing the lowest oscillation amplitudes and the lowest average RMS to reduce the rubber springs stiffness.

To obtain numerical counterevidence, the standard curves for the vertical oscillations (Figure $21 a$ ) and the horizontal oscillations (Figure $21 b$ ) were developed. The

a)

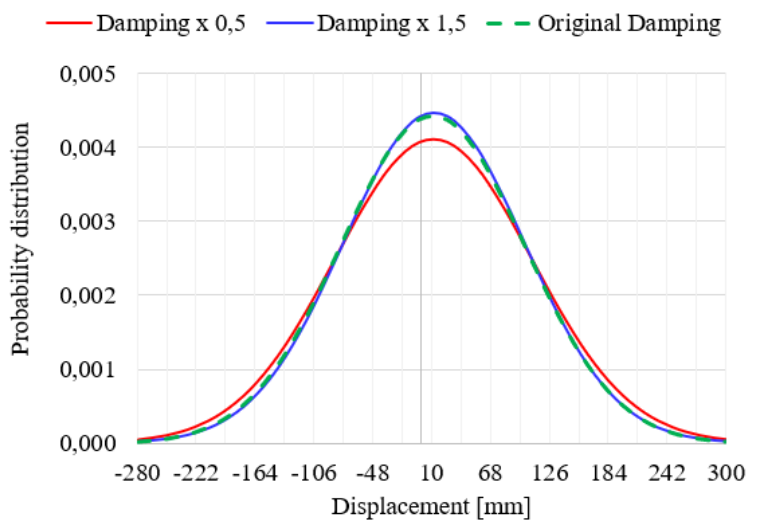

standard curves agree with the average RMS in identifying damping $x 1.5$ and stiffness $\times 0.5$ as the most stable behavior of the spray bar, as the corresponding curves are narrower and "higher" than the others. This indicates that the position of the left end of the bar oscillates closer to the mean. The mean and standard deviation for developments of the normal curves are presented in Table 3.

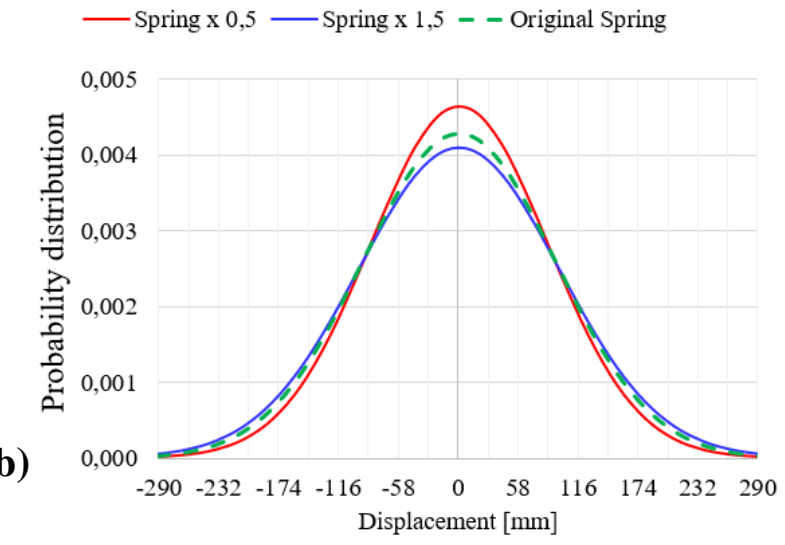

FIGURE 21. Normal distribution for the three levels of damping (a) and stiffness (b) simulated in the FEM.

TABLE 3. FEM results by varying stiffness and damping parameters.

\begin{tabular}{ccccccc}
\hline $\begin{array}{c}\text { Displacement } \\
\text { Variable }\end{array}$ & \multicolumn{3}{c}{$\begin{array}{c}\text { Vertical } \\
\text { Damping of the frame }\end{array}$} & \multicolumn{2}{c}{$\begin{array}{c}\text { Horizontal } \\
\text { Rubber spring stiffness }\end{array}$} \\
\hline Multiplier & x 0,5 & Original (x 1) & x 1,5 & x 0,5 & Original (x 1) \\
Minimum & $-281,99$ & $-242,13$ & $-227,8$ & $-313,23$ & $-315,94$ & $-332,89$ \\
Maximum & 353,76 & 309,8 & 292,75 & 224,57 & 262,12 & 305,68 \\
Average & 11,61 & 11,64 & 12,17 & 0,98 & 0,76 & 0,68 \\
Standard Deviation & 96,86 & 90,29 & 89,19 & 86,07 & 93,26 & 93,31 \\
RMS & 97,53 & 91,02 & 90,00 & 86,06 & 97,30 \\
\hline
\end{tabular}

The standard and average RMS curves are simple means for comparing results and determining the most stable behavior. There is no significant difference in comparing the results presented in Table 3, possibly because the coupled sprayer under study is already a marketed product. Therefore, it is believed that this type of equipment already has some improvement.

Passive suspension can reduce vertical and horizontal oscillation amplitudes but cannot eliminate them. The procedure explored in this study is not limited to sprayers coupled with trapezoidal suspension type "A." It can be applied to other types of boom sprayers in the future.

\section{CONCLUSIONS}

This study determined that the alternate parameter range results in significant improvement. It demonstrated that the horizontal stiffness of the bar influences the oscillations at its extremity. This behavior should be included in simulations of the suspension system. The stiffness is increased by resizing the structure to reduce the bar's horizontal oscillations.

\section{REFERENCES}

Alves AF (2013) Finite elements. The basis of CAE technology. São Paulo, 6 ed.
Bjornsson OH, Wiggers SL, Maagaard J, Terp CI (2013) Dynamic analysis of the intelligent sprayer boom. In: International Conference on Vibration Problems. Lisbon.

Engelen K (2008) Passive damping of flexible spray boom structures. Thesis, Katholieke Universiteit Leuven, Faculteit Ingenieurswetenschappen.

Gohari M, Tahmasebi M, Mailah M, Rahman RA (2017) Vibration suppression of sprayer boom structure using active torque control and iterative learning. Part II: Experimental implementation. Journal of Vibration and control $0(0): 1-11$. DOI: https://doi.org/10.1177/1077546317733907.

Herbst A, Osteroth HJ, Stendel H (2018) A novel method for testing automatic systems for controlling the spray boom height. Biosystems Engineering 174(1):115-125. DOI: https://doi.org/10.1016/j.biosystemseng.2018.06.003.

ISO 14131 (2005) Agricultural sprayers - Boom steadiness - Test methods.

ISO 5008 (2002) Agricultural wheeled tractors and field machinery measurement of whole-body vibration.

Koc C (2015) Structural analysis of field sprayer booms. Journal of Agricultural Sciences 23(1):147-155. 
Langenakens JJ, Clijmans L, Ramon H, Baerdemaeker J (1999) The effects of vertical sprayer boom movements on the uniformity of spray distribution. Journal of Agricultural Engineering Research 74(3):218-291. DOI: https://doi.org/10.1006/jaer.1999.0464.

Machado TM, Reynaldo EF, Taubinger L, Quadros D (2016) Vertical and horizontal oscillation of three models of self-propelled boom sprayers. Brazilian Journal of Agricultural and Environmental Engineering 20(10)941945. DOI: http://dx.doi.org/10.1590/18071929/agriambi.v20n10p941-945.

Manea D, Gidea M, Marin E, Mateescu M (2018) Simulation of mechanical parameters of sprayer boom. Enginnering for rural development 23(1):45-51. DOI: https://doi.org/10.22616/ERDev2018.17.N048.

Pontelli CO, Mucheroni MF, Balthazar JM, Pontes BR (2010) Comparison of two types of passive boom suspension under simulated standardized test track conditions. Engenharia Agricola 30(4):761-775. DOI: https://doi.org/10.1590/S0100-69162010000400020.
Pontelli CO, Mucheroni MF (2009) Model validation of a boom suspension used in trailed sprayers. Minerva Journal - Research \& Technology, 6(2)189-196.

Sobotka SM, Lipinski AJ (2015) A new structure of the stabilization system of a suspended sprayer boom. Agricultural Engineering 1(153):127-132 DOI: http://dx.medra.org/10.14654/ir.2015.153.113.

Stãnescu C, Tudose L, Pop D, Fischer NU (2010) Rolling movement simulation of a 21 boom spray. Buletinul Instititutului Politeehnic Din Iasi. Available: https://www.researchgate.net/publication/290445803. Accessed Nov 12, 2020.

Tahmasebi M, Mailah M, Gohari M, Rahman RA (2018) Vibration suppression of sprayer boom structure using active torque control and iterative learning. Part I: Modelling and control via simulation. Journal of Vibration and Control 24(20)4689-4699. DOI: https://doi.org/10.1177/1077546317733164. 